TOKYO J. MATH.

VOL. 31, No. 2, 2008

\title{
Double Lerch Value Relations and Functional Relations for Witten Zeta Functions
}

\author{
Takashi NAKAMURA
}

Nagoya University

(Communicated by M. Kurihara)

\begin{abstract}
In this paper, we obtain functional relations for Witten zeta functions by using relations of double Lerch values. By these functional relations, we obtain new proofs of known results on the Tornheim double zeta values, the Euler-Zagier double zeta values, and their alternating and character analogues.
\end{abstract}

\section{Introduction}

For $s_{1}, s_{2}, s_{3}, s_{4}, s_{5}, s_{6} \in \mathbf{C}$ and $0<\alpha, \beta, \gamma, \delta, \eta, \theta \leq 1$, we define generalized Witten zeta functions for $\operatorname{sl}(2), \operatorname{sl}(3)$ and $\operatorname{sl}(4)$ by

$$
\begin{aligned}
\zeta_{s l(2)}\left(s_{1} ; \alpha\right) & :=\sum_{n=1}^{\infty} \frac{e^{2 \pi i n \alpha}}{n^{s_{1}}}, \\
\zeta_{s l(3)}\left(s_{1}, s_{2}, s_{3} ; \alpha, \beta, \gamma\right) & :=\sum_{l, m=1}^{\infty} \frac{e^{2 \pi i l \alpha} e^{2 \pi i m \beta} e^{2 \pi i(l+m) \gamma}}{l^{s_{1}} m^{s_{2}}(l+m)^{s_{3}}}, \\
\zeta_{s l(4)}\left(s_{1}, s_{2}, s_{3}, s_{4}, s_{5}, s_{6} ; \alpha, \beta, \gamma, \delta, \eta, \theta\right) & :=\sum_{l, m, n=1}^{\infty} \frac{e^{2 \pi i l \alpha} e^{2 \pi i m \beta} e^{2 \pi i n \gamma} e^{2 \pi i(l+m) \delta} e^{2 \pi i(m+n) \eta} e^{2 \pi i(l+m+n) \theta}}{l^{s_{1}} m^{s_{2}} n^{s_{3}}(l+m)^{s_{4}}(m+n)^{s_{5}(l+m+n)^{s_{6}}}},
\end{aligned}
$$

in the region of convergence. Originally, Zagier [30] defined Witten zeta functions by

$$
\zeta_{g}(s):=\sum_{\rho}(\operatorname{dim} \rho)^{-s}
$$

where $s \in \mathbf{C}$ and $\rho$ runs over all finite dimensional irreducible representations of a certain semisimple Lie algebra $g$. The values of $\zeta_{g}(2 k)$ for $k \in \mathbf{N}$ had been studied by Witten [27] before Zagier's work in order to calculate the volumes of certain moduli spaces. Afterwards, in [3], Gunnells and Sczech gave explicit formula for $\zeta_{s l(n)}(2 k)$. As a generalization of $\zeta_{g}(s)$, Matsumoto [7] defined the Witten zeta function for $g=s o(5)$ of several complex variables

Received August 17, 2007; revised December 3, 2007

2000 Mathematics Subject Classification: 11M41 (Primary)

Key words and phrases: Witten zeta function, functional relation, double zeta, $L$ and Lerch values 
and proved its analytic continuation. Then Matsumoto and Tsumura [11] introduced Witten zeta function of several variables for $g=\operatorname{sl}(n)(n \geq 2)$.

In the case $\alpha=\beta=\gamma=\delta=\eta=\theta=1$, we write them as $\zeta\left(s_{1}\right), \zeta_{s l(3)}\left(s_{1}, s_{2}, s_{3}\right)$ and $\zeta_{s l(4)}\left(s_{1}, s_{2}, s_{3}, s_{4}, s_{5}, s_{6}\right)$, respectively, which coincide with the zeta functions introduced by Matsumoto and Tsumura [11]. Needless to say, $\zeta\left(s_{1}\right)$ is the Riemann zeta function. The function $\zeta_{s l(3)}\left(s_{1}, s_{2}, s_{3}\right)$ is called the Tornheim double zeta function. The values $\zeta_{s l(3)}(a, b, c)$ for $a, b, c \in \mathbf{N}$ were investigated by Tornheim [17] and Mordell [12] in the 1950's. Recently, Tsumura [25] discovered a functional relation for the Tornheim double zeta function. Next the author [13] found a different approach to this problem. The author's expression of functional relation looks different from Tsumura's, but in [8], it is shown that Tsumura's expression coincides with the author's one.

As a triple analogue of $\zeta_{s l(3)}\left(s_{1}, s_{2}, s_{3}\right)$, the function $\zeta_{s l(4)}\left(s_{1}, s_{2}, s_{3}, 0,0, s_{6}\right)$ is called the Mordell-Torheim triple zeta function, continued analytically, and a functional relation has been obtained in [8]. Matsumoto continued $\zeta_{s l(4)}\left(s_{1}, s_{2}, s_{3}, s_{4}, s_{5}, s_{6}\right)$ analytically in [7, Theorem 3]. Afterwards Matsumoto and Tsumura determined its singularities more closely in [11, Theorem 3.5]. Functional relations for $\zeta_{s l(4)}\left(s_{1}, s_{2}, s_{3}, s_{4}, s_{5}, s_{6}\right)$ were proved in Matsumoto and Tsumura [11]. Afterwards, the author [15] showed new proofs of these functional relations and another type of functional relations for $\zeta_{s l(4)}\left(s_{1}, s_{2}, s_{3}, s_{4}, s_{5}, s_{6}\right)$. In [5], Komori, Matsumoto and Tsumura discuss these functions as zeta-functions of root systems. In [6], independently of the present work of the author, they prove functional relations for Witten zeta functions of other Lie algebras.

On the other hand, Euler-Zagier multiple zeta values, the double case of which is written by $\zeta_{s l(3)}(0, a, b)$, have been investigated by a lot of mathematicians (see for example Hoffman's web page). These values are related to knot theory, cohomology of motives and so on. Arakawa and Kaneko defined multiple $L$-values, the double case of which is written by $\zeta_{s l(3)}(0, a, b ; 1, \beta, \gamma), \beta, \gamma \in \mathbf{Q}$. They studied the regularized double shuffle and the derivation relations of multiple $L$-values and gave some applications in [2]. Afterwards Terhune gave some evaluations for double $L$-values in [16].

In this paper, we obtain functional relations for generalized Witten zeta functions. All functional relations for Witten zeta functions proved in this paper are derived from the double Lerch value relation (2.2). We can obtain new functional relations because double Lerch value relation (2.2) deduces the Bernoulli polynomial formula (5.8), which is a key to the proof of functional relations showed in [13], [14] and [15]. By multiplying the double Lerch value relation (2.2) by some functions and integrating the resulting expression, we obtain all functional relations for generalized Witten zeta functions in this paper (see the proofs of Lemma 4.1, Theorems 3.1, 5.3, 5.5, 6.2 and 6.3). Hence we may guess that all of functional relations for Witten zeta functions could be constructed by multiple Lerch value relations. As an evidence, we will show that a lot of known results on double Lerch values and Witten zeta functions are induced from (2.2).

This paper is divided into 6 sections. In Section 2, we prove (2.2). We show the functional relation for $\zeta_{s l(3)}\left(s_{1}, s_{2}, s_{3} ; \alpha, \beta, \gamma\right)$ which is a generalization of [13, Theorem 1.2] and 
[14, Theorem 3.2] in Theorem 3.1. By using Theorem 3.1, we obtain new proofs of Tornheim [17, Theorem 2] (resp. Tsumura [24, Theorem 2] and [19, Theorem 3.4]), in Proposition 3.2 (resp. Proposition 3.3). In Section 4, we obtain functional relations for $\zeta_{s l}(3)\left(s_{1}, s_{2}, s_{3}\right)$ with characters in Lemmas 4.1, 4.2, 4.3 and Theorem 4.4. By these theorems, we can obtain explicit expression for double Lerch values and Tornheim double zeta values with characters. For example, we can obtain new proofs of Terhune [16, Theorem 1] and Tsumura [21, Theorem 3.1], (see Propositions 4.5 and 4.8). In Section 5, we show a functional relation for Witten zeta functions attached to $s o(5)$ in Theorems 5.3 and 5.5. In Section 6, we obtain functional relations (6.1) and (6.2), which are generalizations of [11, Theorem 5.9 and 5.10] and [15, Theorems 2.1 and 2.3]. In Theorem 6.3, we obtain the functional relation (6.3).

\section{Double Lerch values}

We define double Lerch values by

$$
\mathcal{L}(a, b ; x, y):=\lim _{R \rightarrow \infty} \sum_{\substack{R \geq m+n \\ m, n=1}} \frac{e^{2 \pi i m x} e^{2 \pi i n y}}{(m+n)^{a} n^{b}}, \quad a, b \in \mathbf{N}, \quad x, y \in \mathbf{R} .
$$

These and their multiple sum versions have been already defined in Arakawa and Kaneko [2] for the case $x, y \in \mathbf{Q}$ as special cases of their multiple $L$-values. In the case $a \geq 2$, $\mathcal{L}(a, b ; x, y)$ are absolutely convergent. As for the case $a=1$, we have the following criterion.

Proposition 2.1. For $x \in \mathbf{R} \backslash \mathbf{Z}$, the series $\mathcal{L}(1, b ; x, y)$ is uniformly convergent in the wider sense in $(0,1)$.

PROOF. We can obtain this proposition by modifying the proof of [2, Proposition 1.1], which is based on Abel's summation.

For the sake of simplicity, we say that the index set $(a, b ; x, y)$ for which the series $\mathcal{L}(a, b ; x, y)$ is convergent is admissible. The next double Lerch values relation plays an important role in this paper.

THEOREM 2.2. For any admissible index, we have

$$
\begin{aligned}
& \zeta_{s l(2)}(a ; x) \zeta_{s l(2)}(b ; x-y)+(-1)^{b} \mathcal{L}(a, b ; x, y)+(-1)^{a} \mathcal{L}(b, a ; x-y,-y) \\
& =\sum_{j=1}^{a}\left(\begin{array}{c}
a+b-j-1 \\
a-j
\end{array}\right) \zeta_{s l(2)}(a+b-j ; x-y)\left(\zeta_{s l(2)}(j ; y)+(-1)^{j} \zeta_{s l(2)}(j ;-y)\right) \\
& \quad+\sum_{j=1}^{b}\left(\begin{array}{c}
a+b-j-1 \\
b-j
\end{array}\right) \zeta_{s l(2)}(a+b-j ; x)\left(\zeta_{s l(2)}(j ;-y)+(-1)^{j} \zeta_{s l(2)}(j ; y)\right) \\
& \quad-\left(\begin{array}{c}
a+b-1 \\
a
\end{array}\right) \zeta_{s l(2)}(a+b ; x-y)-\left(\begin{array}{c}
a+b-1 \\
b
\end{array}\right) \zeta_{s l(2)}(a+b ; x) .
\end{aligned}
$$


We remark that when $y \rightarrow 0$ the term corresponding to $j=1$ of the first sum on the right-hand side of (2.2) and the term corresponding to $j=1$ of the second sum are cancelled.

PROOF. We use the identity

$$
\begin{aligned}
\frac{(-1)^{b} e^{2 \pi i r x} e^{2 \pi i k y}}{r^{u}(k+r)^{a} k^{b+v}}= & \sum_{j=1}^{b}\left(\begin{array}{c}
a+b-j-1 \\
b-j
\end{array}\right) \frac{(-1)^{j} e^{2 \pi i r x} e^{2 \pi i k y}}{r^{a+b-j+u} k^{j+v}} \\
& +\sum_{j=1}^{a}\left(\begin{array}{c}
a+b-j-1 \\
a-j
\end{array}\right) \frac{e^{2 \pi i r x} e^{2 \pi i k y}}{r^{a+b-j+u}(k+r)^{j} k^{v}}
\end{aligned}
$$

where $k, r \in \mathbf{N}, u, v \in \mathbf{C}, \Re(u)>1$ and $\Re(v)>1$, which follows from Huard, Williams and Zhang [4, (1.12)]. Summing on $k$ and $r$, we have

$$
\begin{aligned}
& (-1)^{b} \zeta_{s l(3)}(u, b+v, a ; x, y, 1) \\
& \quad=\sum_{j=1}^{b}\left(\begin{array}{c}
a+b-j-1 \\
b-j
\end{array}\right)(-1)^{j} \zeta_{s l(2)}(a+b-j+u ; x) \zeta_{s l(2)}(j+v ; y) \\
& \quad+\sum_{j=1}^{a}\left(\begin{array}{c}
a+b-j-1 \\
a-j
\end{array}\right) \zeta_{s l(3)}(a+b-j+u, v, j ; x, y, 1) .
\end{aligned}
$$

By analytic continuation ([14, Theorem 2.1]), we find that the above formula is valid in the case $u=v=0$. We may regard that the formula with $u=v=0$ is a kind of shuffle product formula. Next applying the harmonic product formula

$$
\zeta_{s l(2)}(s ; x-y) \zeta_{s l(2)}(t ; y)=\mathcal{L}(s, t ; x-y, y)+\mathcal{L}(t, s ; y, x)+\zeta_{s l(2)}(s+t ; x)
$$

to the case $u=v=0$ in (2.3), using $\zeta_{s l(3)}(a+b-j, 0, j ; x, y, 1)=\mathcal{L}(j, a+b-j ; y, x)$ and changing the order of sum involving the term $\mathcal{L}(a+b-j, j ; x-y, x)$, we have

$$
\begin{aligned}
& (-1)^{b} \mathcal{L}(a, b ; x, y) \\
& =\sum_{j=1}^{b}\left(\begin{array}{c}
a+b-j-1 \\
b-j
\end{array}\right)(-1)^{j} \zeta_{s l(2)}(a+b-j ; x) \zeta_{s l(2)}(j ; y) \\
& \quad+\sum_{j=1}^{a}\left(\begin{array}{c}
a+b-j-1 \\
a-j
\end{array}\right)\left(\zeta_{s l(2)}(a+b-j ; x-y) \zeta_{s l(2)}(j ; y)-\zeta_{s l(2)}(a+b ; x)\right) \\
& \quad-\sum_{h=0}^{a-1}\left(\begin{array}{c}
b-1+h \\
h
\end{array}\right) \mathcal{L}(b+h, a-h ; x-y, x) .
\end{aligned}
$$


By changing variables and parameters as $x \mapsto x-y$ and $y \mapsto-y$ in the above formula, we have

$$
\begin{aligned}
(-1)^{a} \mathcal{L}(b, a ; x-y,-y) \\
=\sum_{j=1}^{a}\left(\begin{array}{c}
a+b-j-1 \\
a-j
\end{array}\right)(-1)^{j} \zeta_{s l(2)}(a+b-j ; x-y) \zeta_{s l(2)}(j ;-y) \\
\quad+\sum_{j=1}^{b}\left(\begin{array}{c}
a+b-j-1 \\
b-j
\end{array}\right)\left(\zeta_{s l(2)}(a+b-j ; x) \zeta_{s l(2)}(j ;-y)-\zeta_{s l(2)}(a+b ; x-y)\right) \\
\quad-\sum_{h=0}^{b-1}\left(\begin{array}{c}
a-1+h \\
h
\end{array}\right) \mathcal{L}(a+h, b-h ; x, x-y) .
\end{aligned}
$$

By using the shuffle product formula of Arakawa and Kaneko [2, p. 972, (8)] (in [2], this equation is shown for only $x, y \in \mathbf{Q}$. But this formula holds for $x, y \in \mathbf{R}$ by continuity of double Lerch values for admissible indices which can be derived from the uniform convergence in the wider sense in $(0,1)$, and denseness of $\mathbf{Q}$ in $\mathbf{R})$, we have

$$
\begin{aligned}
\zeta_{s l(2)}(a ; x) \zeta_{s l(2)}(b ; x-y)= & \sum_{h=0}^{a-1}\left(\begin{array}{c}
b-1+h \\
h
\end{array}\right) \mathcal{L}(b+h, a-h ; x-y, x) \\
& +\sum_{h=0}^{b-1}\left(\begin{array}{c}
a-1+h \\
h
\end{array}\right) \mathcal{L}(b+h, a-h ; x, x-y),
\end{aligned}
$$

and applying the well-known formula $\sum_{j=1}^{b}\left(\begin{array}{c}a+b-j-1 \\ b-j\end{array}\right)=\left(\begin{array}{c}a+b-1 \\ a\end{array}\right)$, we obtain this theorem.

We write by $K(a, b ; x, y)$ the right hand side of $(2.2)$. In the case $(x, y)=(1,1)$, the next proposition has already been proved by Huard, Williams and Zhang [4, Theorem 1].

Proposition 2.3. Suppose $a+b \in 2 \mathbf{N}+1$, and $(x, y)$ is equal to one of $(1,1)$, $(1 / 2,1),(1,1 / 2)$, or $(1 / 2,1 / 2)$. For any admissible index, we have

$$
\begin{aligned}
2 \mathcal{L}(a, b ; x, y)= & \zeta_{s l(2)}(a ;-x) \zeta_{s l(2)}(b ; x-y)-\zeta_{s l(2)}(a+b ;-y) \\
& -(-1)^{b} \zeta_{s l(2)}(a ; x) \zeta_{s l(2)}(b ; x-y)+(-1)^{b} K(a, b ; x, y) .
\end{aligned}
$$

Proof. Multiplying (2.2) by $(-1)^{b}$, we have

$$
\begin{aligned}
(-1)^{b} \zeta_{s l(2)}(a ; x) \zeta_{s l(2)}(b ; x-y)+\mathcal{L}(a, b ; x, y) \\
+(-1)^{a+b} \mathcal{L}(b, a ; x-y,-y)=(-1)^{b} K(a, b ; x, y) .
\end{aligned}
$$

By summing the above formula and the harmonic product formula (2.4) with changing the variables as $s \mapsto a$ and $t \mapsto b$ and the parameters as $x-y \mapsto-x$ and $y \mapsto x-y$, we can remove $\mathcal{L}(b, a ; x-y,-y)$. In these cases, we have $\mathcal{L}(a, b ; x, y)=\mathcal{L}(a, b ;-x,-y)$. Hence we obtain this proposition. 
REMARK. After completing the present paper, the author noticed that the above Proposition has been also proved by Xia Zhou, Tianxin Cai and D. Bradley [29] independently.

REMARK. By taking $a=b$ in (2.2), we can obtain an explicit evaluation formula for $\mathcal{L}(a, a ; x, 1)$. We can also obtain an explicit evaluation formula for $\mathcal{L}(a, a ; y, 2 y)$ by putting $a=b$ in (2.4).

\section{3. $\operatorname{sl}(3)$}

Our main result in this section is Theorem 3.1 below. The special case $\beta=\gamma=1$ of this theorem was first obtained in Tsumura [25, Theorem 4.5] and [13, Theorem 1.2], and then, the case $\beta=1$ was proved in [14, Theorem 3.2]. Because of (3.2) with the parameter $\beta$, which is proved by (2.2), we can obtain functional relations (4.5), (5.6), (5.9) and (6.3), for example. This is a novel point of the present paper ; we have only obtained the case $\beta=1$,

$$
\begin{gathered}
\zeta_{s l(3)}(a, b, s ; 1,1, \gamma)+(-1)^{b} \zeta_{s l(3)}(b, s, a ; 1, \gamma, 1)+(-1)^{a} \zeta_{s l(3)}(s, a, b ; \gamma, 1,1) \\
=2 \sum_{j=0}^{\max \{a, b\} / 2}\left\{\left(\begin{array}{c}
a+b-2 j-1 \\
a-2 j
\end{array}\right)+\left(\begin{array}{c}
a+b-2 j-1 \\
b-2 j
\end{array}\right)\right\} \\
\quad \times \zeta(2 j) \zeta_{s l(2)}(a+b+s-j ; \gamma),
\end{gathered}
$$

in [13, Theorem 1.2] and [14, Theorem 3.2], since these are based on (5.8), which is weaker than (actually deduced from) (2.2) (see Lemma 5.4). We remark that the function $\zeta_{s l(3)}(s, t, u ; \alpha, \beta, \gamma)$ is continued meromorphically (see [14, Theorem 2.1]).

THEOREM 3.1. For all $a, b \in \mathbf{N}$ and $s \in \mathbf{C}$ except for the singular points, we have

$$
\begin{aligned}
& \zeta_{s l(3)}(a, b, s ; 1, \beta, \gamma)+(-1)^{b} \zeta_{s l(3)}(b, s, a ;-\beta, \gamma, 1)+(-1)^{a} \zeta_{s l(3)}(s, a, b ; \gamma, 1, \beta) \\
& =\sum_{j=1}^{a}\left(\begin{array}{c}
a+b-j-1 \\
a-j
\end{array}\right) \zeta_{s l(2)}(a+b+s-j ; \beta+\gamma)\left(\zeta_{s l(2)}(j ;-\beta)+(-1)^{j} \zeta_{s l(2)}(j ; \beta)\right) \\
& \quad+\sum_{j=1}^{b}\left(\begin{array}{c}
a+b-j-1 \\
b-j
\end{array}\right) \zeta_{s l(2)}(a+b+s-j ; \gamma)\left(\zeta_{s l(2)}(j ; \beta)+(-1)^{j} \zeta_{s l(2)}(j ;-\beta)\right) \\
& \quad-\left(\begin{array}{c}
a+b-1 \\
a
\end{array}\right) \zeta_{s l(2)}(a+b+s ; \beta+\gamma)-\left(\begin{array}{c}
a+b-1 \\
b
\end{array}\right) \zeta_{s l(2)}(a+b+s ; \gamma) .
\end{aligned}
$$

REMARK. When $\beta \rightarrow 0$ the term corresponding to $j=1$ of the first sum on the righthand side of (2.2) and the term corresponding to $j=1$ of the second sum are cancelled. Hence we find that (3.2) coincides with (3.1) when $\beta \rightarrow 0$.

Proof. Firstly, we assume $a, b \geq 2$, and $\Re(s)>1$. Change the parameter as $-y \mapsto \beta$ in (2.2), multiply by $\sum_{n=1}^{\infty} e^{2 \pi i n(\gamma-x)} n^{-s}$ and integrate from 0 to 1 with respect to $x$. Then 
we have

$$
\begin{gathered}
\int_{0}^{1} \sum_{l=1}^{\infty} \frac{e^{2 \pi i l x}}{l^{a}} \sum_{m=1}^{\infty} \frac{e^{2 \pi i m(x+\beta)}}{m^{b}} \sum_{n=1}^{\infty} \frac{e^{2 \pi i n(\gamma-x)}}{n^{s}} d x=\sum_{l, m=1}^{\infty} \frac{e^{2 \pi i m \beta} e^{2 \pi i(l+m) \gamma}}{l^{a} m^{b}(l+m)^{s}}, \\
\int_{0}^{1} \sum_{l, m=1}^{\infty} \frac{e^{2 \pi i m x} e^{-2 \pi i l \beta}}{(m+l)^{a} l^{b}} \sum_{n=1}^{\infty} \frac{e^{2 \pi i n(\gamma-x)}}{n^{s}} d x=\sum_{l, m=1}^{\infty} \frac{e^{-2 \pi i l \beta} e^{2 \pi i m \gamma}}{(l+m)^{a} l^{b} m^{s}} \\
\int_{0}^{1} \sum_{l=1}^{\infty} \frac{e^{2 \pi i l(x+\beta)}}{l^{a+b-j}} \sum_{n=1}^{\infty} \frac{e^{2 \pi i n(\gamma-x)}}{n^{s}} d x=\sum_{l=1}^{\infty} \frac{e^{2 \pi i l(\beta+\gamma)}}{l^{a+b+s-j}}
\end{gathered}
$$

which are $\zeta_{s l(3)}(a, b, s ; 1, \beta, \gamma)$ and $\zeta_{s l(3)}(b, s, a ;-\beta, \gamma, 1)$ on the left-hand side of (3.2) and $\zeta_{s l(2)}(a+b+s-j ; \beta+\gamma)$ on the right-hand side of (3.2). We can also obtain $\zeta_{s l(3)}(s, a, b ; \gamma, 1, \beta)$ and $\zeta_{s l(2)}(a+b+s-j ; \gamma)$, similarly. Hence we have (3.2) in this region.

Next we consider the case $a=1, b \geq 2$. We define $K(a, b, s ; \beta, \gamma)$ by the right-hand side of (3.2). We modify some basic properties in Huard, Williams and Zhang [4, (1.5)] proved by easy computations, for $\alpha, \beta, \gamma \in \mathbf{R}$ and $s, t, u \in \mathbf{C}$ except for the singular points, which are

$$
\left\{\begin{array}{l}
\zeta_{s l(3)}(s, t-1, u+1 ; \alpha, \beta, \gamma)+\zeta_{s l(3)}(s-1, t, u+1 ; \alpha, \beta, \gamma)=\zeta_{s l(3)}(s, t, u ; \alpha, \beta, \gamma) \\
\zeta_{s l(3)}(s, t+1, u-1 ; \alpha, \beta, \gamma)-\zeta_{s l(3)}(s-1, t+1, u ; \alpha, \beta, \gamma)=\zeta_{s l(3)}(s, t, u ; \alpha, \beta, \gamma) \\
\zeta_{s l(3)}(s+1, t, u-1 ; \alpha, \beta, \gamma)-\zeta_{s l(3)}(s+1, t-1, u ; \alpha, \beta, \gamma)=\zeta_{s l(3)}(s, t, u ; \alpha, \beta, \gamma)
\end{array}\right.
$$

By (3.3) and the result in the case $a, b \geq 2$ which we have already shown, we have

$$
\begin{aligned}
K(2, b, s ; \beta, \gamma)= & \zeta_{s l(3)}(2, b, s ; 1, \beta, \gamma)+(-1)^{b} \zeta_{s l(3)}(b, s, 2 ;-\beta, \gamma, 1) \\
& +(-1)^{2} \zeta_{s l(3)}(s, 2, b ; \gamma, 1, \beta) \\
= & \zeta_{s l(3)}(1, b, s+1 ; 1, \beta, \gamma)+(-1)^{b} \zeta_{s l(3)}(b, s+1,1 ;-\beta, \gamma, 1) \\
& +(-1) \zeta_{s l(3)}(s+1,1, b ; \gamma, 1, \beta)+K(2, b-1, s+1 ; \beta, \gamma),
\end{aligned}
$$

for $b \geq 2$. Therefore we have to show

(3.4) $\quad K(2, b, s ; \beta, \gamma)=K(1, b, s+1 ; \beta, \gamma)+K(2, b-1, s+1 ; \beta, \gamma), \quad b \geq 2$.

Especially, we must treat four binomial coefficients on the right-hand side of (3.2). We can see that the first binomial coefficients appear from the formula $\left(\begin{array}{c}2+b-j-1 \\ 2-j\end{array}\right)=\left(\begin{array}{c}1+b-j-1 \\ 1-j\end{array}\right)+$ $\left(\begin{array}{c}2+b-1-j-1 \\ 2-j\end{array}\right)$, which is easily deduced by Pascal's triangle. The other binomial coefficients on the right-hand side of (3.2) appear similarly. Hence we obtain (3.4). We can prove (3.2) for the cases $a \geq 2, b=1$ and $a=b=1$ in the same way.

By (3.2), we obtain the following proposition, which has already been proved by Tornheim. 
Proposition 3.2 ([17, Theorem 2]). For $3 \leq N \in \mathbf{N}$, we have

$$
\zeta_{s l(3)}(1, N-2,1)=\frac{N+1}{2} \zeta(N)-\frac{1}{2} \sum_{j=2}^{N-j} \zeta(j) \zeta(N-j),
$$

$$
\zeta_{s l(3)}(1,1, N-2)=2 \zeta_{s l(3)}(1,0, N-1)=(N-1) \zeta(N)-\sum_{j=2}^{N-j} \zeta(j) \zeta(N-j) .
$$

PROOF. The following proof is a simplified version of [17, Theorem 2]. By (3.3), we have

$$
\zeta_{s l(3)}(j, 0, N-j)+\zeta_{s l(3)}(j-1,1, N-j)=\zeta_{s l(3)}(j, 1, N-j-1) .
$$

Summing on $j$ from 2 to $N-2$, we have

$$
\sum_{j=2}^{N-2} \zeta_{s l(3)}(j, 0, N-j)+\zeta_{s l(3)}(1,1, N-2)=\zeta_{s l(3)}(N-2,1,1) .
$$

From the harmonic product formula, we have

$$
2 \sum_{j=2}^{N-2} \zeta_{s l(3)}(j, 0, N-j)=\sum_{j=2}^{N-2} \zeta(j) \zeta(N-j)-(N-3) \zeta(N) .
$$

Taking $a=b=\beta=\gamma=1$ and $s=N-2$ in (3.2), we obtain

$$
2 \zeta_{s l(3)}(N-2,1,1)-\zeta_{s l(3)}(1,1, N-2)=2 \zeta(N) .
$$

Therefore we have the expilcit evaluation formulae for $\zeta_{s l(3)}(N-2,1,1)$ and $\zeta_{s l(3)}(1,1, N-2)$ from the above three formulae. We can also obtain evaluation formula for $\zeta_{s l(3)}(1,0, N-1)$ by (3.3).

Explicit evaluation formulae for $\zeta_{s l(3)}(a, b, c), \quad \zeta_{s l(3)}(a, b, c ; 1 / 2,1,1)$ and $\zeta_{s l(3)}(a, b, c ; 1,1,1 / 2)$ for $a, b, c \in \mathbf{N}, a+b+c \in 2 \mathbf{N}+1$, have been already proved in Huard, Williams and Zhang [4, Theorem 2] (see also the proof of [13, (3.2)]), Tsumura [24, Theorem 2] and [19, Theorem 3.4], respectively. Afterwards, Xia Zhou, Tianxin Cai and D. Bradley gave evaluation formulae for $\zeta_{s l(3)}(a, b, c ; 1 / 2,1,1), \zeta_{s l(3)}(a, b, c ; 1,1 / 2,1)$ and $\zeta_{s l(3)}(a, b, c ; 1,1,1 / 2)$ by using [29, Corollary 1, Proposition 1, and Equation (5)]. We give new and simple proofs and evaluation formulae for $\zeta_{s l(3)}(a, b, c ; 1 / 2,1,1)$, $\zeta_{s l(3)}(a, b, c ; 1,1 / 2,1)$ and $\zeta_{s l(3)}(a, b, c ; 1,1,1 / 2)$ in the next proposition.

Proposition 3.3 (see [29]). For $a, b, c \in \mathbf{N}, a+b+c \in 2 \mathbf{N}+1$, we have

(3.7) $2 \zeta_{s l(3)}(a, b, c ; 1 / 2,1,1)=(-1)^{a} K(c, a, b ; 1 / 2,1)+(-1)^{b} K(b, c, a ; 1,1 / 2)$,

(3.8) $2 \zeta_{s l(3)}(a, b, c ; 1,1 / 2,1)=(-1)^{a} K(c, a, b ; 1,1 / 2)+(-1)^{b} K(b, c, a ; 1 / 2,1 / 2)$,

(3.9) $2 \zeta_{s l(3)}(a, b, c ; 1,1,1 / 2)=(-1)^{a} K(c, a, b ; 1 / 2,1 / 2)+(-1)^{b} K(b, c, a ; 1 / 2,1)$. 
Proof. Putting $\beta=1 / 2, \gamma=1$, and $\beta=1, \gamma=1 / 2$, and $\beta=\gamma=1 / 2$ in (3.2), and changing variables, we have the following six formulae;

$$
\begin{aligned}
& \quad \zeta_{s l(3)}(b, c, a ; 1,1 / 2,1)+(-1)^{c} \zeta_{s l(3)}(c, a, b ; 1 / 2,1,1)+(-1)^{b} \zeta_{s l(3)}(a, b, c ; 1,1,1 / 2) \\
& \quad=K(b, c, a ; 1 / 2,1), \\
& \zeta_{s l(3)}(c, a, b ; 1,1 / 2,1)+(-1)^{a} \zeta_{s l(3)}(a, b, c ; 1 / 2,1,1)+(-1)^{c} \zeta_{s l(3)}(b, c, a ; 1,1,1 / 2) \\
& \quad=K(c, a, b ; 1 / 2,1), \\
& \zeta_{s l(3)}(b, c, a ; 1,1,1 / 2)+(-1)^{c} \zeta_{s l(3)}(c, a, b ; 1,1 / 2,1)+(-1)^{b} \zeta_{s l(3)}(a, b, c ; 1 / 2,1,1) \\
& \quad=K(b, c, a ; 1,1 / 2), \\
& \zeta_{s l(3)}(c, a, b ; 1,1,1 / 2)+(-1)^{a} \zeta_{s l(3)}(a, b, c ; 1,1 / 2,1)+(-1)^{c} \zeta_{s l(3)}(b, c, a ; 1 / 2,1,1) \\
& \quad=K(c, a, b ; 1,1 / 2), \\
& \zeta_{s l(3)}(b, c, a ; 1 / 2,1,1)+(-1)^{c} \zeta_{s l(3)}(c, a, b ; 1,1,1 / 2)+(-1)^{b} \zeta_{s l(3)}(a, b, c ; 1,1 / 2,1) \\
& \quad=K(b, c, a ; 1 / 2,1 / 2), \\
& \zeta_{s l(3)}(c, a, b ; 1 / 2,1,1)+(-1)^{a} \zeta_{s l(3)}(a, b, c ; 1,1,1 / 2)+(-1)^{c} \zeta_{s l(3)}(b, c, a ; 1,1 / 2,1) \\
& \quad=K(c, a, b ; 1 / 2,1 / 2) .
\end{aligned}
$$

Multiplying the second formula by $(-1)^{a}$ and the third formula by $(-1)^{b}$, and summing the resulting formulae, we can remove $\zeta_{s l(3)}(c, a, b ; 1,1 / 2,1)$ and $\zeta_{s l(3)}(b, c, a ; 1,1,1 / 2)$ since $a+b+c \in 2 \mathbf{N}+1$. Hence we obtain (3.7). Similarly, we can also obtain (3.8) (resp. (3.9)), by multiplying the fourth (resp. sixth) formula by $(-1)^{a}$ and fifth (resp. first) formula by $(-1)^{b}$ and summing the resulting formulae. Needless to say, we can prove (3.7) by (3.8) and $\zeta_{s l(3)}(a, b, c ; 1 / 2,1,1)=\zeta_{s l(3)}(b, a, c ; 1,1 / 2,1)$. But we adopt the above proof intentionally, since we want to show the symmetry in the proof.

\section{4. $\quad s l(3)$ with characters}

We define $L^{\varphi}(s ; \alpha)$ and $L_{s l(3)}^{\varphi, \chi, \psi}\left(s_{1}, s_{2}, s_{3} ; \alpha, \beta, \gamma\right)$ by

$$
\begin{gathered}
L^{\varphi}(s ; \alpha):=\sum_{n=1}^{\infty} \frac{e^{2 \pi i n \alpha} \varphi(n)}{n^{s}}, \\
L_{s l(3)}^{\varphi, \chi, \psi}\left(s_{1}, s_{2}, s_{3} ; \alpha, \beta, \gamma\right):=\sum_{l, m=1}^{\infty} \frac{e^{2 \pi i l \alpha} e^{2 \pi i m \beta} e^{2 \pi i(l+m) \gamma} \varphi(l) \chi(m) \psi(l+m)}{l^{s_{1}} m^{s_{2}}(l+m)^{s_{3}}},
\end{gathered}
$$

where $\varphi, \chi$ and $\psi$ are primitive Dirichlet characters of conductor $g, f$ and $h$, respectively. Next we consider analytic continuation for $L_{s l(3)}^{\varphi, \chi, \psi}\left(s_{1}, s_{2}, s_{3} ; \alpha, \beta, \gamma\right)$. In the case $\psi=1$ (trivial character) and $\alpha=\beta=\gamma=1$, Wu has continued this function analytically in [28, 
Theorem 1]. We denote the Gauss sum by $\tau(\chi):=\sum_{l=1}^{f} \chi(l) e^{2 \pi i l / f}$, and recall the wellknown formula

$$
e^{2 \pi i n \beta} \chi(n)=\frac{e^{2 \pi i n \beta}}{\tau(\bar{\chi})} \sum_{l=1}^{f} \bar{\chi}(l) e^{2 \pi i n l / f},
$$

which holds for non-principal and primitive characters. By this formula, $L_{s l(3)}^{\varphi, \chi, \psi}\left(s_{1}, s_{2}\right.$, $\left.s_{3} ; \alpha, \beta, \gamma\right)$ can be written as

$$
\begin{aligned}
& \frac{1}{\tau(\bar{\varphi}) \tau(\bar{\chi}) \tau(\bar{\psi})} \sum_{j_{1}=1}^{g} \sum_{j_{2}=1}^{f} \sum_{j_{3}=1}^{h} \bar{\varphi}\left(j_{1}\right) \bar{\chi}\left(j_{2}\right) \bar{\psi}\left(j_{3}\right) \\
& \times \sum_{l, m=1}^{\infty} \frac{e^{2 \pi i l\left(\alpha+j_{1} / g\right)} e^{2 \pi i m\left(\beta+j_{2} / f\right)} e^{2 \pi i(l+m)\left(\gamma+j_{3} / h\right)}}{l^{s_{1}} m^{s_{2}}(l+m)^{s_{3}}}
\end{aligned}
$$

in the region of convergence (see [9, Section 2]). Since the existence of the analytic continuation of the infinite series on the right-hand side has been shown in ([14, Theorem 2.1]), we also obtain the existence of the analytic continuation for $L_{s l(3)}^{\varphi, \chi, \psi}\left(s_{1}, s_{2}, s_{3} ; \alpha, \beta, \gamma\right)$. Firstly, we will show a functional relation with a Dirichlet character $\psi$.

Lemma 4.1. For all $a, b \in \mathbf{N}$ and $s \in \mathbf{C}$ except for the singular points, we have

$$
\begin{aligned}
L_{s l(3)}^{1,1, \psi}(a, b, s ; 1, \beta, \gamma)+(-1)^{b} L_{s l(3)}^{1, \psi, 1}(b, s, a ;-\beta, \gamma, 1)+(-1)^{a} L_{s l(3)}^{\psi, 1,1}(s, a, b ; \gamma, 1, \beta) \\
=\sum_{j=1}^{a}\left(\begin{array}{c}
a+b-j-1 \\
a-j
\end{array}\right) L^{\psi}(a+b+s-j ; \beta+\gamma)\left(\zeta_{s l(2)}(j ;-\beta)+(-1)^{j} \zeta_{s l(2)}(j ; \beta)\right) \\
\quad+\sum_{j=1}^{b}\left(\begin{array}{c}
a+b-j-1 \\
b-j
\end{array}\right) L^{\psi}(a+b+s-j ; \gamma)\left(\zeta_{s l(2)}(j ; \beta)+(-1)^{j} \zeta_{s l(2)}(j ;-\beta)\right) \\
\quad-\left(\begin{array}{c}
a+b-1 \\
a
\end{array}\right) L^{\psi}(a+b+s ; \beta+\gamma)-\left(\begin{array}{c}
a+b-1 \\
b
\end{array}\right) L^{\psi}(a+b+s ; \gamma) .
\end{aligned}
$$

PROOF. Firstly, we assume $a, b \geq 2$, and $\Re(s)>1$. Change the parameter as $-y \mapsto \beta$ in (2.2), multiply by $\sum_{n=1}^{\infty} \psi(n) e^{2 \pi i n(\gamma-x)} n^{-s}$ and integrate from 0 to 1 with respect to $x$. Then we have

$$
\begin{gathered}
\int_{0}^{1} \sum_{l=1}^{\infty} \frac{e^{2 \pi i l x}}{l^{a}} \sum_{m=1}^{\infty} \frac{e^{2 \pi i m(x+\beta)}}{m^{b}} \sum_{n=1}^{\infty} \frac{\psi(n) e^{2 \pi i n(\gamma-x)}}{n^{s}} d x \\
=\sum_{l, m=1}^{\infty} \frac{\psi(l+m) e^{2 \pi i m \beta} e^{2 \pi i(l+m) \gamma}}{l^{a} m^{b}(l+m)^{s}}
\end{gathered}
$$

which is the first term on the left-hand side of (4.2). We obtain the other terms of (4.2), similarly. Hence we have (4.2) in this region. In the case $a, b \leq 2$, we can prove this lemma similarly to the proof of Theorem 3.1. 
In the case of Dirichlet characters with conductor 4, the following lemma seems to coincide with Tsumura [23, Proposition 4.3], while in the general cases, it seems to coincide with Tsumura [26, Theorem 3.1], though we have not checked rigorously (see also [22, Theorem 4.7]).

LEMma 4.2. Suppose $\psi$ is a Dirichlet character, $\chi$ is a non-principal and primitive Dirichlet character of conductor $f$. For all $a, b \in \mathbf{N}$ and $s \in \mathbf{C}$ except for the singular points, we have

$$
\begin{aligned}
& L_{s l(3)}^{1, \chi, \psi}(a, b, s ; 1, \beta, \gamma)+(-1)^{b} \chi(-1) L_{s l(3)}^{\chi, \psi, 1}(b, s, a ;-\beta, \gamma, 1) \\
& +(-1)^{a} L_{s l(3)}^{\psi, 1, \chi}(s, a, b ; \gamma, 1, \beta) \\
& =\frac{1}{\tau(\bar{\chi})} \sum_{l=1}^{f} \bar{\chi}(m) \sum_{j=1}^{a}\left(\begin{array}{c}
a+b-j-1 \\
a-j
\end{array}\right) L^{\psi}(a+b+s-j ; \beta+\gamma+l / f) \\
& \times\left(\zeta_{s l(2)}(j ;-\beta-l / f)+(-1)^{j} \zeta_{s l(2)}(j ; \beta+l / f)\right) \\
& +\sum_{j=1}^{b}\left(\begin{array}{c}
a+b-j-1 \\
b-j
\end{array}\right) L^{\psi}(a+b+s-j ; \gamma) \\
& \times\left(L^{\chi}(j ; \beta)+(-1)^{j} \chi(-1) L^{\chi}(j ;-\beta)\right) \\
& -\left(\begin{array}{c}
a+b-1 \\
a
\end{array}\right) L^{\chi \psi}(a+b+s ; \beta+\gamma) \text {. }
\end{aligned}
$$

Proof. Change the parameter as $\beta \mapsto l / f+\beta$ in (4.2) and multiply these formulae by $\bar{\chi}(l) / \tau(\bar{\chi})$ for each $l$. Summing the resulting formulae on $l$ from 1 to $f$, and using

$$
e^{2 \pi i n \beta} \sum_{l=1}^{f} \bar{\chi}(l) e^{2 \pi i(-l) n / f}=e^{2 \pi i n \beta} \chi(-n) \tau(\chi)=e^{2 \pi i n \beta} \chi(-1) \sum_{l=1}^{f} \bar{\chi}(l) e^{2 \pi i l n / f},
$$

we obtain this lemma.

Hereafter we also use simplied symbols

$$
L_{s l(3)}^{\varphi, \chi, \psi}\left(s_{1}, s_{2}, s_{3}\right):=L_{s l(3)}^{\varphi, \chi, \psi}\left(s_{1}, s_{2}, s_{3} ; 1,1,1\right), \quad L^{\chi}(s):=L^{\chi}(s ; 1) .
$$

Taking parameters $-\beta=\gamma=m / g$ in (4.2) and multiplying these formulae by $\bar{\varphi}(m) / \tau(\bar{\varphi})$ for each $m$, and summing the resulting formulae on $m$ from 1 to $g$, we obtain the next lemma.

Lemma 4.3. Suppose $\psi$ is a Dirichlet character, $\varphi$ is a non-principal and primitive Dirichlet character of conductor $g$. For all $a, b \in \mathbf{N}$ and $s \in \mathbf{C}$ except for the singular points, 
we have

$$
\begin{aligned}
& L_{s l(3)}^{\varphi, 1, \psi}(a, b, s)+(-1)^{b} L_{s l(3)}^{1, \psi, \varphi}(b, s, a)+(-1)^{a} \varphi(-1) L_{s l(3)}^{\psi, \varphi, 1}(s, a, b) \\
& =\sum_{j=1}^{a}\left(\begin{array}{c}
a+b-j-1 \\
a-j
\end{array}\right) L^{\psi}(a+b+s-j)\left(L^{\varphi}(j)+(-1)^{j} \varphi(-1) L^{\varphi}(j)\right) \\
& \quad+\frac{1}{\tau(\bar{\varphi})} \sum_{m=1}^{g} \bar{\varphi}(k) \sum_{j=1}^{b}\left(\begin{array}{c}
a+b-j-1 \\
b-j
\end{array}\right) L^{\psi}(a+b+s-j ; m / g) \\
& \quad \times\left(\zeta_{s l(2)}(j ;-m / g)+(-1)^{j} \zeta_{s l(2)}(j ; m / g)\right) \\
& -\left(\begin{array}{c}
a+b-1 \\
b
\end{array}\right) L^{\varphi \psi}(a+b+s) .
\end{aligned}
$$

Take parameters $-\beta=\gamma=m / g$ in (4.3) and multiply these formulae by $\bar{\varphi}(m) / \tau(\bar{\varphi})$ for each $m$. Summing the resulting formulae on $m$ from 1 to $g$ for each $m$, we obtain the following theorem.

THEOREM 4.4. Suppose $\psi$ is a Dirichlet character, $\chi$ and $\varphi$ are non-principal and primitive Dirichlet characters of conductor $f$ and $g$, respectively. For all $a, b \in \mathbf{N}$ and $s \in \mathbf{C}$ except for the singular points, we have

$$
\begin{aligned}
L_{s l(3)}^{\varphi, \chi, \psi}(a, b, s)+(-1)^{b} \chi(-1) L_{s l(3)}^{\chi, \psi, \varphi}(b, s, a)+(-1)^{a} \varphi(-1) L_{s l(3)}^{\psi, \varphi, \chi}(s, a, b) \\
=\frac{1}{\tau(\bar{\chi})} \sum_{l=1}^{f} \bar{\chi}(l) \sum_{j=1}^{a}\left(\begin{array}{c}
a+b-j-1 \\
a-j
\end{array}\right) L^{\psi}(a+b+s-j ; l / f) \\
\quad \times\left(L^{\varphi}(j ;-l / f)+(-1)^{j} \varphi(-1) L^{\varphi}(j ; l / f)\right) \\
+\frac{1}{\tau(\bar{\varphi})} \sum_{m=1}^{g} \bar{\varphi}(m) \sum_{j=1}^{b}\left(\begin{array}{c}
a+b-j-1 \\
b-j
\end{array}\right) L^{\psi}(a+b+s-j ; m / g) \\
\quad \times\left(L^{\chi}(j ;-m / g)+(-1)^{j} \chi(-1) L^{\chi}(j ; m / g)\right) .
\end{aligned}
$$

Next we will show explicit evaluation formulae for double Lerch values with characters, similarly to Proposition 2.3. We define $D_{1}(a, b, s ; 1,1, \psi)$ by the right-hand side of (4.2) with $\beta=\gamma=1, D_{2}(a, b, s ; 1, \chi, \psi)$ by the right-hand side of (4.3) with $\beta=\gamma=1$, $D_{3}(a, b, s ; \varphi, 1, \psi)$ and $D_{4}(a, b, s ; \varphi, \chi, \psi)$ by the right-hand side of (4.4) and (4.5), respectively.

PROPOSITION 4.5. If $\chi$ is non-principal and primitive and $\chi(-1)=(-1)^{a+b-1}$, then for any admissible index, we have

$$
\begin{aligned}
2 L_{s l(3)}^{1,1, \chi}(0, a, b)= & \left(1-(-1)^{a}\right) \zeta(a) L^{\chi}(b)-L^{\chi}(a+b) \\
& +(-1)^{a} D_{2}(a, b, 0 ; 1, \chi, 1)
\end{aligned}
$$




$$
\begin{aligned}
2 L_{s l(3)}^{1, \chi, 1}(0, b, a)= & \left(1+(-1)^{a}\right) \zeta(a) L^{\chi}(b)-L^{\chi}(a+b) \\
& -(-1)^{a} D_{2}(a, b, 0 ; 1, \chi, 1)
\end{aligned}
$$

If $\varphi$ and $\chi$ are non-principal and primitive and $\chi(-1) \varphi(-1)=(-1)^{a+b-1}$, then for any admissible index, we have

$$
\begin{aligned}
2 L_{s l(3)}^{1, \varphi, \chi}(0, a, b)= & \left(1-(-1)^{a} \varphi(-1)\right) L^{\varphi}(a) L^{\chi}(b)-L^{\varphi \chi}(a+b) \\
& +(-1)^{a} \varphi(-1) D_{4}(a, b, 0 ; \varphi, \chi, 1) .
\end{aligned}
$$

PROOF. We recall the harmonic product formula

$$
L_{s l(3)}^{1, \varphi, \chi}(0, a, b)+L_{s l(3)}^{1, \chi, \varphi}(0, b, a)+L^{\varphi \chi}(a+b)=L^{\varphi}(a) L^{\chi}(b) .
$$

By putting $s=0$ in (4.3), multiplying it by $(-1)^{a}$, adding the resulting expression and (4.9), we can remove $L_{s l(3)}^{1, \chi, 1}(0, b, a)$. Hence we have (4.6). By (4.9) and (4.6), we obtain (4.7). By putting $s=0$ in (4.5), multiplying it by $(-1)^{a} \varphi(-1)$, and summing the resulting expression and (4.9), we obtain (4.8).

For $l$ and $m$, we let $R_{l, m}$ be the set of all polynomials in convergent series of the form $\sum_{n=1}^{\infty} e^{2 \pi i n \xi_{l}} n^{-j}$ (where $\xi_{l}$ 's are $l$ th roots of unit and $j \in \mathbf{N}$ ) with $\mathbf{Q}\left(e^{2 \pi i / m}\right)$ coefficients. By (4.1) and Proposition 4.5, we can immediately obtain the following proposition, which has already proved by Terhune.

PROPOSITION 4.6 ([16, Theorem 1]). Let $\chi$ and $\varphi$ be Dirichlet characters of conductor $f$ and $h$, respectively. Set $m=\operatorname{lcm}(f, h)$ and $M=\operatorname{lcm}(f, h, \phi(f), \phi(h))$, where $1 \mathrm{~cm}$ means the least common multiple and $\phi$ is the Euler totient function. For any admissible index, if $\chi(-1) \varphi(-1)=(-1)^{a+b+1}$, then $L_{s l(3)}^{1, \varphi, \chi}(0, a, b) \in R_{M, m}$.

Next we will show explicit evaluation formulae for the Tornheim double zeta function with characters similarly to Proposition 3.3.

PROPOSITION 4.7. If $\varphi, \chi$ and $\psi$ are non-principal and primitive character and $\varphi(-1) \chi(-1) \psi(-1)=(-1)^{a+b+c+1}$, then we have

$$
\begin{aligned}
2 L_{s l(3)}^{\varphi, \chi, \psi}(a, b, c)= & (-1)^{b} \chi(-1) D_{4}(b, c, a ; \chi, \psi, \varphi) \\
& +(-1)^{a} \varphi(-1) D_{4}(c, a, b ; \psi, \varphi, \chi) .
\end{aligned}
$$

PROOF. By changing parameters and characters in (4.5), we have

$$
\begin{aligned}
& L_{s l(3)}^{\chi, \psi, \varphi}(b, c, a)+(-1)^{c} \psi(-1) L_{s l(3)}^{\psi, \varphi, \chi}(c, a, b)+(-1)^{b} \chi(-1) L_{s l(3)}^{\varphi, \chi, \psi}(a, b, c) \\
& \quad=D_{4}(b, c, a ; \chi, \psi, \varphi), \\
& L_{s l(3)}^{\psi, \varphi, \chi}(c, a, b)+(-1)^{a} \varphi(-1) L_{s l(3)}^{\varphi, \chi, \psi}(a, b, c)+(-1)^{c} \psi(-1) L_{s l(3)}^{\chi, \psi, \varphi}(b, c, a) \\
& \quad=D_{4}(c, a, b ; \psi, \varphi, \chi) .
\end{aligned}
$$


In the case of $\varphi(-1) \chi(-1) \psi(-1)=(-1)^{a+b+c+1}$, we can remove $L_{s l(3)}^{\chi, \psi, \varphi}(b, c, a)$ and $L_{s l(3)}^{\psi, \varphi, \chi}(c, a, b)$ by multiplying the former formula by $(-1)^{b} \chi(-1)$ and the latter formula by $(-1)^{a} \varphi(-1)$. Hence we obtain (4.10).

We can obtain the following proposition, which should be compared with Tsumura [21, Theorem 3.1], similarly to the above.

PROPOSITION 4.8. If $\psi$ is non-principal and primitive and $\psi(-1)=(-1)^{a+b+c+1}$, then we have

(4.11) $2 L_{s l(3)}^{1,1, \psi}(a, b, c)=(-1)^{b} D_{2}(b, c, a ; 1, \psi, 1)+(-1)^{a} D_{1}(c, a, b ; 1,1, \psi)$,

(4.12) $2 L_{s l(3)}^{1, \psi, 1}(a, b, c)=(-1)^{b} \psi(-1) D_{3}(b, c, a ; \psi, 1,1)+(-1)^{a} D_{1}(c, a, b ; 1,1, \psi)$.

If $\chi$ and $\psi$ are non-principal and primitive and $\chi(-1) \psi(-1)=(-1)^{a+b+c+1}$, then we have (4.13) $2 L_{s l(3)}^{1, \chi, \psi}(a, b, c)=(-1)^{b} \chi(-1) D_{4}(b, c, a ; \chi, \psi, 1)+(-1)^{a} D_{3}(c, a, b ; \psi, 1, \chi)$,

$$
\begin{aligned}
2 L_{s l(3)}^{\chi, \psi, 1}(a, b, c)= & (-1)^{b} \psi(-1) D_{3}(b, c, a ; \psi, 1, \chi) \\
& +(-1)^{a} \chi(-1) D_{2}(c, a, b ; 1, \chi, \psi) .
\end{aligned}
$$

5. $\operatorname{so}(5)$

For $s, t, u \in \mathbf{C}, \Re(s) \geq 1, \Re(t) \geq 1, \Re(u) \geq 1$, we define four functions;

$$
\begin{aligned}
T_{E 1}(s, t, u) & :=\sum_{l, m=1}^{\infty} \frac{1}{(2 l)^{s} m^{t}(2 l+m)^{u}}, \quad T_{E 2}(s, t, u):=\sum_{l, m=1}^{\infty} \frac{1}{l^{s}(2 m)^{t}(l+2 m)^{u}}, \\
T_{O O}(s, t, u) & :=\sum_{l, m=1}^{\infty} \frac{1}{(2 l-1)^{s}(2 m-1)^{t}(2 l+2 m-2)^{u}}, \\
T_{A E}(s, t, u) & :=\sum_{\substack{l, m, n=1 \\
2 n=l+m}}^{\infty} \frac{1}{l^{s} m^{t} n^{u}}=2^{-s-t} \zeta_{s l(3)}(s, t, u)+2^{u} T_{O O}(s, t, u) .
\end{aligned}
$$

PROPOSITION 5.1. For $\Re(s) \geq 1, \Re(t) \geq 1$, and $\Re(u) \geq 1$, we have

$$
\begin{gathered}
T_{E 1}(s, t, u)=2^{-1} \zeta_{s l(3)}(s, t, u)+2^{-1} \zeta_{s l(3)}(s, t, u ; 1 / 2,1,1) \\
T_{E 2}(s, t, u)=2^{-1} \zeta_{s l(3)}(s, t, u)+2^{-1} \zeta_{s l(3)}(s, t, u ; 1,1 / 2,1), \\
T_{O O}(s, t, u)=\left(2^{-1}-2^{-s-t-u}\right) \zeta_{s l(3)}(s, t, u)+2^{-1} \zeta_{s l(3)}(s, t, u ; 1,1,1 / 2), \\
T_{A E}(s, t, u)=2^{u-1} \zeta_{s l(3)}(s, t, u)+2^{u-1} \zeta_{s l(3)}(s, t, u ; 1,1,1 / 2)
\end{gathered}
$$

The functions $T_{E 1}(s, t, u), T_{E 2}(s, t, u), T_{O O}(s, t, u)$ and $T_{A E}(s, t, u)$ are continued meromorphically, and the above formulae hold for all $s, t, u \in \mathbf{C}$ except for the singularities. Moreover, in the case $a, b, c \in \mathbf{N}, a+b+c \in 2 \mathbf{N}+1, T_{E 1}(a, b, c), T_{E 2}(a, b, c), T_{O O}(a, b, c)$ and $T_{A E}(a, b, c)$ are polynomials in $\{\zeta(k) \mid 2 \leq k \leq a+b+c\}$ with rational coefficients. 
Proof. Assume that $\Re(s) \geq 1, \mathfrak{R}(t) \geq 1$ and $\Re(u) \geq 1$. The first, second and fourth formulae are obvious. By the definition of $\zeta_{s l(3)}(s, t, u ; \alpha, \beta, \gamma)$ and $T_{O O}(s, t, u)$, we immediately obtain

$$
\begin{aligned}
& \zeta_{s l(3)}(s, t, u)+\zeta_{s l(3)}(s, t, u ; 1 / 2,1,1)+\zeta_{s l(3)}(s, t, u ; 1,1 / 2,1)+\zeta_{s l(3)}(s, t, u ; 1,1,1 / 2) \\
& \quad=2^{2-s-t-u} \zeta_{s l(3)}(s, t, u) \\
& \zeta_{s l(3)}(s, t, u)-\zeta_{s l(3)}(s, t, u ; 1 / 2,1,1)-\zeta_{s l(3)}(s, t, u ; 1,1 / 2,1)+\zeta_{s l(3)}(s, t, u ; 1,1,1 / 2) \\
& \quad=4 T_{O O}(s, t, u) .
\end{aligned}
$$

By summing the above formulae, we obtain the third formula of this proposition. All the righthand side of the above formulae in this proposition are continued meromorphically by analytic continuation of $\zeta_{s l(3)}(s, t, u ; \alpha, \beta, \gamma)$ (see [14, Theorem 2.1]). Therefore by these formulae we can define $T_{E 1}(s, t, u), T_{E 2}(s, t, u), T_{O O}(s, t, u)$ and $T_{A E}(s, t, u)$ for $s, t, u \in \mathbf{C}$ except for singularities. By using the formulae in this proposition and Proposition 3.3, we obtain the last assertion of this proposition.

For $s, t, u, v \in \mathbf{C}$, we define the Witten zeta function attached to $s o(5)$ by

$$
\zeta_{s o(5)}(s, t, u, v):=\sum_{l, m=1}^{\infty} \frac{1}{l^{s} m^{t}(l+m)^{u}(l+2 m)^{v}},
$$

in the region of convergence. We recall that Matsumoto defined this function and continued analytically in [7, Theorem 3]. The following proposition has already been shown by Tsumura. We can show this proposition in a simpler way, since the evaluation formulae of $T_{E 1}(a, b, c), T_{E 2}(a, b, c)$ and $\zeta_{s l(3)}(a, b, c)$ for $a+b+c \in 2 \mathbf{N}+1$ are obtained much simpler.

Proposition 5.2 ([20, Theorem]). Suppose that $a, b, c, d \in \mathbf{N}$ and $a+b+c+d \in$ $2 \mathbf{N}+1$. Then $\zeta_{\text {so(5) }}(a, b, c, d)$ can be expressed as a rational linear combination of products of Riemann's zeta values at positive integers.

ProOF. We quote [20, (10)],

$$
\begin{aligned}
\zeta_{\text {so }(5)}(a, b, c, d)= & (-1)^{c} \sum_{j=1}^{c}\left(\begin{array}{c}
c+d-j-1 \\
c-j
\end{array}\right)(-1)^{j} \zeta_{\text {so(5) }}(a, b+c+d-j, j, 0) \\
& +(-1)^{c} \sum_{j=1}^{d}\left(\begin{array}{c}
c+d-j-1 \\
d-j
\end{array}\right)(-1)^{j} \zeta_{\text {so }(5)}(a, b+c+d-j, 0, j) .
\end{aligned}
$$

Hence we obtain this proposition by Propositions 3.3 and 5.1.

Next we will prove a functional relation for two complex variables. 
THEOREM 5.3. For $a, b \in \mathbf{N}$ and $s, t \in \mathbf{C}$ except for singular points, we have

$$
\begin{aligned}
& \zeta_{\text {so(5) }}(a, b, s, t)+(-1)^{b} \zeta_{\text {so }(5)}(t, b, s, a)+(-1)^{a} \zeta_{\text {so }(5)}(a, s, b, t) \\
& =\sum_{j=1}^{a}\left(\begin{array}{c}
a+b-j-1 \\
a-j
\end{array}\right)\left(T_{A E}(j, t, a+b+s-j)\right. \\
& \left.\quad+2^{a+b+s-j}(-1)^{j} T_{E 1}(a+b+s-j, j, t)\right) \\
& \quad+\sum_{j=1}^{b}\left(\begin{array}{c}
a+b-j-1 \\
b-j
\end{array}\right)\left(\zeta_{s l(3)}(a+b+s-j, j, t)\right. \\
& \quad-\left(\begin{array}{c}
a+b-1 \\
a
\end{array}\right) 2^{-t} \zeta(a+b+s+t)-\left(\begin{array}{c}
a+b-1 \\
b
\end{array}\right) \zeta(a+b+s+t) .
\end{aligned}
$$

ProOF. Put $\beta=\gamma=x$ in (3.2), multiply by $\sum_{n=1}^{\infty} e^{-2 \pi i n x} n^{-t}$ and integrate from 0 to 1 with respect to $x$. For $\Re(s)>1$ and $\Re(t)>1$, we have

$$
\begin{gathered}
\int_{0}^{1} \sum_{l, m=1}^{\infty} \frac{e^{2 \pi i l x} e^{2 \pi i m(2 x)}}{l^{a} m^{b}(l+m)^{s}} \sum_{n=1}^{\infty} \frac{e^{-2 \pi i n x}}{n^{t}} d x=\sum_{l, m=1}^{\infty} \frac{1}{l^{a} m^{b}(l+m)^{s}(l+2 m)^{t}} \\
\int_{0}^{1} \sum_{l=1}^{\infty} \frac{e^{2 \pi i l(2 x)}}{l^{a+b+s-j}} \sum_{m=1}^{\infty} \frac{e^{-2 \pi i m x}}{m^{j}} \sum_{n=1}^{\infty} \frac{e^{-2 \pi i n x}}{n^{t}} d x=\sum_{l, m, n=1}^{\infty} \frac{1}{l^{a+b+s-j} m^{j} n^{t}}, \\
\int_{0}^{1} \sum_{l=1}^{\infty} \frac{e^{2 \pi i l(2 x)}}{l^{a+b+s-j}} \sum_{m=1}^{\infty} \frac{e^{2 \pi i m x}}{m^{j}} \sum_{n=1}^{\infty} \frac{e^{-2 \pi i n x}}{n^{t}} d x=\sum_{l, m, n=1}^{\infty} \frac{1}{l^{a+b+s-j} m^{j}(2 l+m)^{t}}, \\
\int_{0}^{1} \sum_{l=1}^{\infty} \frac{e^{2 \pi i l(2 x)}}{l^{a+b+s}} \sum_{n=1}^{\infty} \frac{e^{-2 \pi i n x}}{n^{t}} d x=\sum_{l=1}^{\infty} \frac{1}{l^{a+b+s}(2 l)^{t}},
\end{gathered}
$$

which are $\zeta_{s o(5)}(a, b, s, t), T_{A E}(j, t, a+b+s-j), T_{E 1}(a+b+s-j, j, t)$ and $2^{-t} \zeta(a+$ $b+s+t)$ in (5.6). The other terms of (5.6) are obtained similarly. By analytic continuation for $\zeta_{s l(3)}(s, t, u ; \alpha, \beta, \gamma)$ and $\zeta_{s o(5)}(s, t, u, v)$ (see [14, Theorem 2.1] and [7, Theorem 3]), we have (5.6) except for the singular points. 
LEMMA 5.4. For any admissible index, we have

$$
\begin{aligned}
\left(\zeta_{s l(2)}(c ; x)\right. & \left.+(-1)^{c} \zeta_{s l(2)}(c ;-x)\right)\left(\zeta_{s l(2)}(d ; x)+(-1)^{d} \zeta_{s l(2)}(d ;-x)\right) \\
& +\left((-1)^{c}+(-1)^{d}\right) \zeta(c+d) \\
= & 2 \sum_{j=0}^{c}\left(\begin{array}{c}
c+d-2 j-1 \\
c-2 j
\end{array}\right) \zeta(2 j) \\
& \times\left(\zeta_{s l(2)}(c+d-2 j ; x)+(-1)^{c+d} \zeta_{s l(2)}(c+d-2 j ;-x)\right) \\
& +2 \sum_{j=0}^{d}\left(\begin{array}{c}
c+d-2 j-1 \\
d-2 j
\end{array}\right) \zeta(2 j) \\
& \times\left(\zeta_{s l(2)}(c+d-2 j ; x)+(-1)^{c+d} \zeta_{s l(2)}(c+d-2 j ;-x)\right)
\end{aligned}
$$

PROOF. By the harmonic product formula, we have

$$
\begin{aligned}
\left(\zeta_{s l(2)}(c ; x)+(-1)^{c} \zeta_{s l(2)}(c ;-x)\right)\left(\zeta_{s l(2)}(d ; x)+(-1)^{d} \zeta_{s l(2)}(d ;-x)\right) \\
=\zeta_{s l(2)}(c ; x) \zeta_{s l(2)}(d ; x)+(-1)^{d}(\mathcal{L}(c, d ; x, 0)+\mathcal{L}(d, c ;-x, 0)+\zeta(c+d)) \\
\quad+(-1)^{c}(\mathcal{L}(c, d ;-x, 0)+\mathcal{L}(d, c ; x, 0)+\zeta(c+d)) \\
\quad+(-1)^{c+d} \zeta_{s l(2)}(c ;-x) \zeta_{s l(2)}(d ;-x) .
\end{aligned}
$$

Using (2.2) and $\zeta(0)=-1 / 2$, we obtain this lemma.

REMARK. We denote by $B_{j}(x)$ the Bernoulli polynomial of order $j$ defined by

$$
\frac{t e^{x t}}{e^{t}-1}=\sum_{j=0}^{\infty} B_{j}(x) \frac{t^{j}}{j !}, \quad|t|<2 \pi .
$$

By (5.7), we can immediately prove Carlitz's formula [1, p. 276, 19.(b)], for $p+q \geq 2$, which is

$$
\begin{aligned}
B_{p}(x) B_{q}(x)= & \sum_{k=0}^{\max (p, q) / 2}\left\{p\left(\begin{array}{c}
q \\
2 k
\end{array}\right)+q\left(\begin{array}{c}
p \\
2 k
\end{array}\right)\right\} \frac{B_{2 k} B_{p+q-2 k}(x)}{p+q-2 k} \\
& -(-1)^{p} \frac{p ! q !}{(p+q) !} B_{p+q} .
\end{aligned}
$$

This formula is the key to the proof of [13, Theorem 1.2], which means that a product of Bernoulli polynomials is a linear combination of Bernoulli polynomials. We may regard that this is a special case of a well-known fact, that is, the set of multiple zeta or $L$-values are closed with respect to the operations of the harmonic product and the shuffle product. variable.

At the end of this section, we show the following functional relations for one complex 
THEOREM 5.5. For $a, b, c \in \mathbf{N}$ and $s \in \mathbf{C}$ except for singular points, we have (5.9)

$$
\begin{aligned}
\zeta_{s o(5)}( & a, b, s, c)+(-1)^{b} \zeta_{\text {so }(5)}(c, b, s, a) \\
& +(-1)^{a} \zeta_{\text {so }(5)}(a, s, b, c)+(-1)^{b+c} \zeta_{\text {so }(5)}(c, s, b, a) \\
= & 2 \sum_{d=0}^{a}\left(\begin{array}{c}
a+b-d-1 \\
a-d
\end{array}\right) \sum_{j=0}^{\max (c, d) / 2}\left\{\left(\begin{array}{c}
c+d-2 j-1 \\
c-2 j
\end{array}\right)+\left(\begin{array}{c}
c+d-2 j-1 \\
d-2 j
\end{array}\right)\right\} \\
& \times 2^{2 j-c-d} \zeta(2 j) \zeta(a+b+c+s-2 j) \\
& +2 \sum_{d=0}^{b}(-1)^{d}\left(\begin{array}{c}
a+b-j-1 \\
b-d
\end{array}\right) \sum_{j=0}^{\max (c, d) / 2}\left\{\left(\begin{array}{c}
c+d-2 j-1 \\
c-2 j
\end{array}\right)+\left(\begin{array}{c}
c+d-2 j-1 \\
d-2 j
\end{array}\right)\right\} \\
& \times \zeta(2 j) \zeta(a+b+c+s-2 j) .
\end{aligned}
$$

Proof. Firstly, we assume $\mathfrak{R}(s)>1$. Taking parameters $\beta=\gamma=-x$ in (3.2), multiplying by the series $\left(\sum_{n=1}^{\infty} e^{2 \pi i n x} n^{-c}+(-1)^{c} \sum_{n=1}^{\infty} e^{-2 \pi i n x} n^{-c}\right)$, and integrating from 0 to 1 with respect to $x$, we obtain

$$
\begin{gathered}
\int_{0}^{1} \sum_{l, m=1}^{\infty} \frac{e^{-2 \pi i l x} e^{-2 \pi i(2 m) x}}{l^{a} m^{b}(l+m)^{s}} \sum_{n=1}^{\infty} \frac{e^{2 \pi i n x}}{n^{c}} d x=\sum_{l, m=1}^{\infty} \frac{1}{l^{a} m^{b}(l+m)^{s}(l+2 m)^{c}}, \\
\int_{0}^{1} \sum_{m=1}^{\infty} \frac{e^{-2 \pi i m(2 x)}}{m^{a+b+s-d}}\left(\sum_{n=1}^{\infty} \frac{e^{2 \pi i n x}}{n^{c+d-2 j}}+(-1)^{c+d} \sum_{n=1}^{\infty} \frac{e^{-2 \pi i n x}}{n^{c+d-2 j}}\right) d x \\
=\sum_{m=1}^{\infty} \frac{1}{m^{a+b+s-d}(2 m)^{c+d-2 j}},
\end{gathered}
$$

which are $\zeta_{s o(5)}(a, b, s, c)$ and $2^{2 j-c-d} \zeta(2 j) \zeta(a+b+c+s-2 j)$ in (5.9). Changing the order of summation and integration is justified as follows. We can change the order by the absolute convergence in the case of $c \geq 2$. We recall the well-known formula

$$
\sum_{n=1}^{\infty} \frac{e^{2 \pi i n x}}{n}=-\log (2 \sin \pi x)-i \pi B_{1}(x), \quad 0<x<1 .
$$

We define $f(x)$ by the right-hand side of (5.10). By (5.10), for a sufficiently large integer $N$, there exists an $M>0$ such that

$$
\left|\sum_{n=1}^{N} \frac{e^{2 \pi i n x}}{n}\right| \leq|f(x)|+M, \quad 0<x<1 .
$$

From this fact and the well-known formula

$$
\int_{0}^{1} \log (2 \sin \pi x) d x=\log 2-\log 2=0,
$$


we see that we can use Lebegsue's bounded convergence theorem. The other terms of (5.9) are obtained similarly. By (5.7), $\zeta(0)=-1 / 2$ and the argument similar to the proof of Theorem 5.3 , we obtain this theorem.

6. $\operatorname{sl}(4)$

We define the function

$$
Z(a, b, s, t, u ; \beta, \gamma, \delta, \eta):=\sum_{\substack{l, m, n, k=1 \\ l+m=n+k}}^{\infty} \frac{e^{2 \pi i m \beta} e^{2 \pi i n \gamma} e^{2 \pi i k \delta} e^{2 \pi i(l+m) \eta}}{l^{a} m^{b} n^{s} k^{t}(l+m)^{u}} .
$$

LEMMA 6.1. For $a, b \in \mathbf{N}, s, t, u \in \mathbf{C}, \Re(s)>1, \Re(t)>1, \Re(u)>1$, we have

$$
\begin{aligned}
Z(a, b, s, t, u ; \alpha, \gamma, \delta, \eta)= & \zeta_{s l(3)}(a+s, b+t, u ; \gamma, \beta+\delta, \eta) \\
& +\zeta_{s l(4)}(a, 0, t, s, b, u ; 1,1, \delta, \gamma, \beta, \eta) \\
& +\zeta_{s l(4)}(b, 0, s, t, a, u ; \beta, 1, \gamma, \delta, 1, \eta)
\end{aligned}
$$

PROOF. In this region, we have

$$
\begin{aligned}
Z(a, b, s, t, u ; \alpha, \gamma, \delta, \eta) & =\sum_{h=1}^{\infty} \sum_{\substack{l, m=1 \\
l+m=h}}^{\infty} \frac{e^{2 \pi i m \beta}}{l^{a} m^{b}} \sum_{\substack{n, k=1 \\
n+k=h}}^{\infty} \frac{e^{2 \pi i n \gamma} e^{2 \pi i k \delta} e^{2 \pi i h \eta}}{n^{s} k^{t} h^{u}} \\
& =\sum_{l, n=1}^{\infty} \frac{1}{l^{a} n^{s}} \sum_{h>\max \{l, n\}} \frac{e^{2 \pi i(h-l) \beta} e^{2 \pi i n \gamma} e^{2 \pi i(h-n) \delta} e^{2 \pi i h \eta}}{(h-l)^{b}(h-n)^{t} h^{u}} .
\end{aligned}
$$

We separate the right-hand side of the above formula as $\sum_{l=n}+\sum_{l<n}+\sum_{l>n}$. In the case $l=n$, we have

$$
\sum_{l=n}=\sum_{n=1}^{\infty} \sum_{h>n} \frac{e^{2 \pi i n \gamma} e^{2 \pi i(h-n)(\beta+\delta)} e^{2 \pi i h \eta}}{n^{a+s}(h-n)^{b+t} h^{u}}=\zeta_{s l(3)}(a+s, b+t, u ; \gamma, \beta+\delta, \eta) .
$$

By putting $n=l+j$ and $h=l+j+k$, we have

$$
\begin{aligned}
\sum_{l<n} & =\sum_{l, j, k=1}^{\infty} \frac{e^{2 \pi i(j+k) \beta} e^{2 \pi i(l+j) \gamma} e^{2 \pi i k \delta} e^{2 \pi i(l+j+k) \eta}}{l^{a}(l+j)^{s}(j+k)^{b} k^{t}(l+j+k)^{u}} \\
& =\zeta_{s l(4)}(a, 0, t, s, b, u ; 1,1, \delta, \gamma, \beta, \eta)
\end{aligned}
$$

By putting $l=n+j$ and $h=n+j+k$, we have

$$
\begin{aligned}
\sum_{l>n} & =\sum_{n, j, k=1}^{\infty} \frac{e^{2 \pi i k \beta} e^{2 \pi i n \gamma} e^{2 \pi i(j+k) \delta} e^{2 \pi i(n+j+k) \eta}}{(n+j)^{a} n^{s} k^{b}(j+k)^{t}(n+j+k)^{u}} \\
& =\zeta_{s l(4)}(b, 0, s, t, a, u ; \beta, 1, \gamma, \delta, 1, \eta)
\end{aligned}
$$

Therefore by these formulae, we obtain this lemma. 
In the case $\beta=1$, the next theorem coincides with [15, Theorem 2.1 and Theorem 2.3]. Therefore it also contains [15, Theorem 3.4] which is a functional relation for $\zeta_{s l(4)}(a, b, c, 0,0, s)$.

THEOREM 6.2. For $a, b \in \mathbf{N}, s, t, u \in \mathbf{C}, \mathfrak{R}(s)>1, \mathfrak{R}(t)>1, \mathfrak{R}(u)>1$, we have

$$
\begin{aligned}
\zeta_{s l(4)} & (a, b, t, s, 0, u ; 1, \beta, \delta, \gamma, 1, \eta)+(-1)^{b} \zeta_{s l(4)}(b, s, t, a, u, 0 ;-\beta, \gamma, \delta, 1, \eta, 1) \\
& +(-1)^{a} \zeta_{s l(4)}(a, s, t, b, u, 0 ; 1, \gamma, \delta, \beta, \eta, 1) \\
= & \sum_{j=1}^{a}\left(\begin{array}{c}
a+b-j-1 \\
a-j
\end{array}\right) \zeta_{s l(3)}(a+b+s-j, t, u ; \beta+\gamma, \delta, \eta) \\
& \times\left(\zeta_{s l(2)}(j ;-\beta)+(-1)^{j} \zeta_{s l(2)}(j ; \beta)\right) \\
& +\sum_{j=1}^{b}\left(\begin{array}{c}
a+b-j-1 \\
b-j
\end{array}\right) \zeta_{s l(3)}(a+b+s-j, t, u ; \gamma, \delta, \eta) \\
& \times\left(\zeta_{s l(2)}(j ; \beta)+(-1)^{j} \zeta_{s l(2)}(j ;-\beta)\right) \\
& -\left(\begin{array}{c}
a+b-1 \\
a
\end{array}\right) \zeta_{s l(3)}(a+b+s, t, u ; \beta+\gamma, \delta, \eta) \\
& -\left(\begin{array}{c}
a+b-1 \\
b
\end{array}\right) \zeta_{s l(3)}(a+b+s, t, u ; \gamma, \delta, \eta)
\end{aligned}
$$

and

$$
\begin{aligned}
Z(a, b, s, t, u ; \beta, \gamma, \delta, \eta)+(-1)^{b} \zeta_{s l(4)}(b, t, u, 0, s, a ;-\beta, \delta, \eta, 1, \gamma, 1) \\
\quad+(-1)^{a} \zeta_{s l(4)}(a, u, t, 0, s, b ; 1, \eta, \delta, 1, \gamma, \beta) \\
=\sum_{j=1}^{a}\left(\begin{array}{c}
a+b-j-1 \\
a-j
\end{array}\right) \zeta_{s l(3)}(t, u, a+b+s-j ; \delta, \eta, \beta+\gamma) \\
\quad \times\left(\zeta_{s l(2)}(j ;-\beta)+(-1)^{j} \zeta_{s l(2)}(j ; \beta)\right) \\
\quad+\sum_{j=1}^{b}\left(\begin{array}{c}
a+b-j-1 \\
b-j
\end{array}\right) \zeta_{s l(3)}(t, u, a+b+s-j ; \delta, \eta, \gamma) \\
\quad \times\left(\zeta_{s l(2)}(j ; \beta)+(-1)^{j} \zeta_{s l(2)}(j ;-\beta)\right) \\
\quad-\left(\begin{array}{c}
a+b-1 \\
a
\end{array}\right) \zeta_{s l(3)}(t, u, a+b+s ; \delta, \eta, \beta+\gamma) \\
\quad-\left(\begin{array}{c}
a+b-1 \\
b
\end{array}\right) \zeta_{s l(3)}(t, u, a+b+s ; \delta, \eta, \gamma) .
\end{aligned}
$$

PROOF. Change the parameter as $x \mapsto x+\gamma$ in (3.2), multiply by two functions $\sum_{n=1}^{\infty} e^{2 \pi i n(x+\delta)} n^{-t}$ and $\sum_{k=1}^{\infty} e^{2 \pi i k(\eta-x)} k^{-u}$ and integrate from 0 to 1 with respect to $x$. 
We have

$$
\begin{gathered}
\int_{0}^{1} \sum_{l, m=1}^{\infty} \frac{e^{2 \pi i m \beta} e^{2 \pi i(l+m)(x+\gamma)}}{l^{a} m^{b}(l+m)^{s}} \sum_{n=1}^{\infty} \frac{e^{2 \pi i n(x+\delta)}}{n^{t}} \sum_{k=1}^{\infty} \frac{e^{2 \pi i k(\eta-x)}}{k^{u}} d x \\
=\sum_{l, m, n=1}^{\infty} \frac{e^{2 \pi i m \beta} e^{2 \pi i n \delta} e^{2 \pi i(l+m) \gamma} e^{2 \pi i(l+m+n) \eta}}{l^{a} m^{b} n^{t}(l+m)^{s}(l+m+n)^{u}}, \\
\int_{0}^{1} \sum_{l, m=1}^{\infty} \frac{e^{-2 \pi i l \beta} e^{2 \pi i m(x+\gamma)}}{l^{b} m^{s}(l+m)^{a}} \sum_{n=1}^{\infty} \frac{e^{2 \pi i n(x+\delta)}}{n^{t}} \sum_{k=1}^{\infty} \frac{e^{2 \pi i k(\eta-x)}}{k^{u}} d x \\
=\sum_{l, m, n=1}^{\infty} \frac{e^{-2 \pi i l \beta} e^{2 \pi i m \gamma} e^{2 \pi i n \delta} e^{2 \pi i(m+n) \eta}}{l^{b} m^{s} n^{t}(l+m)^{a}(m+n)^{u}}, \\
\int_{0}^{1} \sum_{l=1}^{\infty} \frac{e^{2 \pi i l(x+\beta+\gamma)} \sum^{a+b+s-j} \sum_{n=1}^{\infty} \frac{e^{2 \pi i n(x+\delta)}}{n^{t}} \sum_{k=1}^{\infty} \frac{e^{2 \pi i k(\eta-x)}}{k^{u}} d x}{l^{a+b+s}} d x \\
\sum_{l, n=1}^{\infty} \frac{e^{2 \pi i l(\beta+\gamma)} e^{2 \pi i n \delta} e^{2 \pi i(l+n) \eta}}{l^{a+b+s-j} n^{t}(l+n)^{u}},
\end{gathered}
$$

which are $\zeta_{s l(4)}(a, b, t, s, 0, u ; 1, \beta, \delta, \gamma, 1, \eta), \quad \zeta_{s l(4)}(b, s, t, a, u, 0 ;-\beta, \gamma, \delta, 1, \eta, 1)$ and $\zeta_{s l(3)}(a+b+s-j, t, u ; \beta+\gamma, \delta, \eta)$ in (6.1). The other terms of (6.1) are obtained in the same way. Next change the parameter as $x \mapsto x+\gamma$ in (3.2), multiply by $\sum_{n=1}^{\infty} e^{2 \pi i n(\delta-x)} n^{-t}$ and $\sum_{k=1}^{\infty} e^{2 \pi i n(\eta-x)} k^{-u}$ and integrate from 0 to 1 with respect to $x$. We obtain

$$
\begin{gathered}
\int_{0}^{1} \sum_{l, m=1}^{\infty} \frac{e^{2 \pi i m \beta} e^{2 \pi i(l+m)(x+\gamma)}}{l^{a} m^{b}(l+m)^{s}} \sum_{n=1}^{\infty} \frac{e^{2 \pi i n(\delta-x)}}{n^{t}} \sum_{k=1}^{\infty} \frac{e^{2 \pi i k(\eta-x)}}{k^{u}} d x \\
=\sum_{\substack{l, m, n, k=1 \\
l+m=n+k}}^{\infty} \frac{e^{2 \pi i m \beta} e^{2 \pi i n \gamma} e^{2 \pi i k \delta} e^{2 \pi i(l+m) \eta}}{l^{a} m^{b} n^{s} k^{t}(l+m)^{u}}, \\
\int_{0}^{1} \sum_{l, m=1}^{\infty} \frac{e^{-2 \pi i l \beta} e^{2 \pi i m(x+\gamma)}}{l^{b} m^{s}(l+m)^{a}} \sum_{n=1}^{\infty} \frac{e^{2 \pi i n(\delta-x)}}{n^{t}} \sum_{k=1}^{\infty} \frac{e^{2 \pi i k(\eta-x)}}{k^{u}} d x \\
=\sum_{l, k, n=1}^{\infty} \frac{e^{-2 \pi i l \beta} e^{2 \pi i(n+k) \gamma} e^{2 \pi i n \delta} e^{2 \pi i k \eta}}{l^{b}(n+k)^{s}(l+n+k)^{a} n^{t} k^{u}}, \\
\int_{0}^{1} \sum_{l=1}^{\infty} \frac{e^{2 \pi i l(x+\beta+\gamma)} \sum^{a+b+s-j} \sum_{n=1}^{\infty} \frac{e^{2 \pi i n(\delta-x)}}{n^{t}} \sum_{k=1}^{\infty} \frac{e^{2 \pi i k(\eta-x)}}{k^{u}} d x}{\sum_{n, k=1}^{\infty} \frac{e^{2 \pi i l(n+k)(\beta+\gamma)} e^{2 \pi i n \delta} e^{2 \pi i k \eta}}{(n+k)^{a+b+s-j} n^{t} k^{u}},}
\end{gathered}
$$


which are $Z(a, b, s, t, u ; \beta, \gamma, \delta, \eta), \zeta_{s l(4)}(b, t, u, 0, s, a ;-\beta, \delta, \eta, 1, \gamma, 1)$ and $\zeta_{s l(3)}(t, u$, $a+b+s-j ; \delta, \eta, \gamma)$ in (6.2). The other terms of (6.2) are obtained similarly. Hence we obtain (6.1) and (6.2).

The following functional relation holds for three complex variables. In [3, Proposition 8.5], Gunnells and Sczech gave explicit formula for $\zeta_{s l(4)}(2 k, 2 k, 2 k, 2 k, 2 k, 2 k)$. In [5, Theorem 4.4], Komori, Matsumoto and Tsumura proved this functional relation in the case $a, b, c \in 2 \mathbf{N}$ and $\gamma=\delta=\eta=1$. These results should be compared with the next theorem.

THEOREM 6.3. For $a, b, c \in \mathbf{N}, s, t, u \in \mathbf{C}, \Re(s)>1, \Re(t)>1, \Re(u)>1$, we have

$$
\begin{aligned}
\zeta_{s l(4)} & (a, b, t, s, c, u ; 1,1, \delta, \gamma, 1, \eta)+(-1)^{b} \zeta_{s l(4)}(s, b, c, a, t, u ; \gamma, 1,1,1, \delta, \eta) \\
+ & (-1)^{a} \zeta_{s l(4)}(a, s, t, b, u, c ; 1, \gamma, \delta, 1, \eta, 1) \\
+ & (-1)^{b+c} \zeta_{s l(4)}(s, t, c, u, b, a ; \gamma, \delta, 1, \eta, 1,1) \\
= & 2 \sum_{d=0}^{a}\left(\begin{array}{c}
a+b-d-1 \\
a-d
\end{array}\right) \sum_{j=0}^{\max (c, d) / 2}\left\{\left(\begin{array}{c}
c+d-2 j-1 \\
c-2 j
\end{array}\right)+\left(\begin{array}{c}
c+d-2 j-1 \\
d-2 j
\end{array}\right)\right\} \\
& \times \zeta(2 j) \zeta_{s l(3)}(a+b+s-d, t, u+c+d-2 j ; \gamma, \delta, \eta) \\
+ & 2 \sum_{d=0}^{b}(-1)^{d}\left(\begin{array}{c}
a+b-d-1 \\
b-d
\end{array}\right) \\
& \times \sum_{j=0}^{\max (c, d) / 2}\left\{\left(\begin{array}{c}
c+d-2 j-1 \\
c-2 j
\end{array}\right)+\left(\begin{array}{c}
c+d-2 j-1 \\
d-2 j
\end{array}\right)\right\} \\
& \times \zeta(2 j) \zeta_{s l(3)}(a+b+s-d, t+c+d-2 j, u ; \gamma, \delta, \eta) .
\end{aligned}
$$

ProOF. Change parameters as $\beta \mapsto-x$ and $\delta \mapsto \delta-x$ in (6.1), multiply by $\left(\sum_{h=1}^{\infty} e^{2 \pi i h x} h^{-c}+(-1)^{c} \sum_{h=1}^{\infty} e^{-2 \pi i h x} h^{-c}\right)$, and integrate from 0 to 1 with respect to $x$. We have

$$
\begin{gathered}
\int_{0}^{1} \sum_{l, m, n=1}^{\infty} \frac{e^{-2 \pi i m x} e^{2 \pi i n(\delta-x)} e^{2 \pi i(l+m) \gamma} e^{2 \pi i(l+m+n) \eta}}{l^{a} m^{b} n^{t}(l+m)^{s}(l+m+n)^{u}} \sum_{h=1}^{\infty} \frac{e^{2 \pi i h x}}{h^{c}} d x \\
=\sum_{l, m, n=1}^{\infty} \frac{e^{2 \pi i n \delta} e^{2 \pi i(l+m) \gamma} e^{2 \pi i(l+m+n) \eta}}{l^{a} m^{b} n^{t}(l+m)^{s}(m+n)^{c}(l+m+n)^{u}} \\
\int_{0}^{1} \sum_{l, m, n=1}^{\infty} \frac{e^{2 \pi i l x} e^{2 \pi i m \gamma} e^{2 \pi i n(\delta-x)} e^{2 \pi i(m+n) \eta}}{l^{b} m^{s} n^{t}(l+m)^{a}(m+n)^{u}} \sum_{h=1}^{\infty} \frac{e^{2 \pi i h x}}{h^{c}} d x \\
=\sum_{l, m, h=1}^{\infty} \frac{e^{2 \pi i m \gamma} e^{2 \pi i(h+l) \delta} e^{2 \pi i(m+l+h) \eta}}{l^{b} m^{s}(l+h)^{t}(l+m)^{a}(m+l+h)^{u} h^{c}}
\end{gathered}
$$


which are $\zeta_{s l(4)}(a, b, t, s, c, u ; 1,1, \delta, \gamma, 1, \eta)$ and $\zeta_{s l(4)}(s, b, c, a, t, u ; \gamma, 1,1,1, \delta, \eta)$ in (6.3). By (5.7) and the manner similar to the proof of Theorem 5.5, we can obtain this theorem.

Acknowledgments. The author thanks Professors Kohji Matsumoto and Hirofumi Tsumura for useful advice. The author is supported by JSPS Research Fellowship for Young Scientist (JSPS Research Fellow DC2).

\section{References}

[1] T. M. Apostol, Introduction to Analytic Number Theory, Springer, 1976.

[2 ] T. Arakawa and M. Kaneko, On multiple $L$-values, J. Math. Soc. Japan 56 (2004), no. 4, 967-991.

[3] E. GunNELls and R. SCZECH, Evaluation of Dedekind sums, Eisenstein cocycles, and special values of L-functions. Duke Math. J. 118 (2003), no. 2, 229-260.

[4] J. G. Huard, K. S. Williams and Z. Y. Zhang, On Tornheim's double series, Acta Arith. 75 (1996), 239-252.

[ 5 ] Y. Komori, K. Matsumoto and H. Tsumura, Zeta-functions of root systems, to appear in Conference on L-functions, L. Weng and M. Kaneko (eds.), World Scientific, 2007.

[ 6 ] Y. Komori, K. MATSUMOto and H. Tsumura, On Witten multiple zeta-functions associated with semisimple Lie algebras III, preprint.

[ 7 ] K. Matsumoto, On Mordell-Tornheim and other multiple zeta-functions, in Proceedings of the Session in Analytic Number Theory and Diophantine Equations, 17 pp., Bonner Math. Schriften, 360, Univ. Bonn, Bonn, 2003.

[ 8 ] K. Matsumoto, T. Nakamura, H. Ochiai and H. Tsumura, On value-relations, functional relations and singularities of Mordell-Tornheim and related triple zeta-functions, preprint.

[ 9 ] K. Matsumoto, T. Nakamura and H. Tsumura, Functional relations and special values of MordellTornheim triple zeta-functions and L-functions, to appear in Proc. Amer. Math. Soc..

[10] K. Matsumoto and H. Tsumura, A new method of producing functional relations among multiple zeta functions, to appear in Quart. J. Math.

[11] K. Matsumoto and H. Tsumura, On Witten multiple zeta-functions associated with semisimple Lie algebras I, Ann. Inst. Fourier 56 (2006), no. 5, 1457-1504.

[12] L. J. Mordell, On the evaluation of some multiple series, J. London Math. Soc. 33 (1958), 368-371.

[13] T. NAKAMURA, A functional relations for the Tornheim double zeta function, Acta Arith 125 (2006), 257-263.

[14] T. NAKAMURA, Double Lerch series and their functional relations, to appear in Aequationes Mathematicae.

[15] T. NAKAMURA, Functional relations related to Witten zeta functions, preprint.

[16] D. Terhune, Evaluation of double $L$-values, J. Number Theory 105 (2004), no. 2, 275-301.

[17] L. ToRnheIm, Harmonic double series, Amer. J. Math. 72 (1950), 303-314.

[18] H. Tsumura, On alternating analogues of Tornheim's double series, Proc. Amer. Math. Soc. 131 (2003), no. $12,3633-3641$.

[19] H. TSUmurA, Evaluation formulae for Tornheim's type of alternating double series, Math. Comp. 73 (2004), no. 245, 251-258.

[20] H. Tsumura, On Witten's type of zeta values attached to SO(5), Arch. Math. (Basel) 82 (2004), no. 2, $147-152$.

[21] H. Tsumura, On evaluation formulae for double $L$-values, Bull. Austral. Math. Soc. 70 (2004), no. 2, 213221.

[22] H. Tsumura, Certain functional relations for the double harmonic series related to the double Euler numbers, J. Aust. Math. Soc. 79 (2005), no. 3, 319-333. 
[23] H. Tsumura, On some functional relations between Mordell-Tornheim double $L$-functions and Dirichlet $L$-functions, J. Number Theory 120 (2006), 161-178.

[24] H. TSUmURA, On alternating analogues of Tornheim's double series II, preprint.

[25] H. Tsumura, On functional relations between the Mordell-Tornheim double zeta functions and the Riemann zeta function," Math. Proc. Camb. Phil. Soc. 142, (2007) 395-405.

[26] H. Tsumura, Certain functional relations for double $L$-functions, preprint.

[27] E. Witten, On quantum gauge theories in two dimensions, Comm. Math. Phys. 141 (1991), no. 1, $153-209$.

[28] Maoxiang WU, On analytic continuation of Mordell-Tornheim and Apostol-Vu L-functions, (in Japanese), Master Thesis, Nagoya Univercity, 2003.

[29] Xia Zhou, Tianxin Cai and D. Bradley, Signed q-Analogs of Tornheim's Double Series, to appear in Proceedings of the American Mathematical Society.

[30] D. Zagier, Values of zeta functions and their applications, First Eupopean Congress of Math., Paris, vol. II, Progress in Math. 120, Birkhäuser 1994, 497-512.

Present Address:

FACULTY OF MATHEMATICS,

KYUSHU UNIVERSITY,

6-10-1 HAKOZAKI, HigASHI-KU, FUKUOKA, 812-8581 JAPAN. 